\title{
Effects of Vibration on Dynamic and Stabiliser Muscle Activities During the Press Up
}

\author{
Dan Robbins ${ }^{1,2, *}$, Shane Bone ${ }^{1}$, Mark Chapman ${ }^{1}$, Mark Goss-Sampson ${ }^{1}$ \\ ${ }^{1}$ Centre for Sports Science, University of Greenwich, Medway, Kent, ME4 6AZ, UK \\ ${ }^{2}$ Division of Sports Therapy, University of Bedfordshire, Luton, LU1 3JU \\ *Corresponding Author: Daniel.robbins@beds.ac.uk
}

Copyright (C) 2014 Horizon Research Publishing All rights reserved.

\begin{abstract}
A pilot study investigating the influence of vibration during press ups on myoelectrical activity of both dynamic and stabiliser muscles. Nine male participants $(21.1$ \pm 3.4 years, $1.68 \pm 0.03 \mathrm{~m}, 72.3 \pm 9.5 \mathrm{~kg}$ ), performed press ups under 3 conditions: without vibration, low amplitude and frequency vibration $(30 \mathrm{~L} ; 1.2 \mathrm{~mm}, 30 \mathrm{~Hz}$ ) high amplitude and frequency vibration $(40 \mathrm{H} ; 1.9 \mathrm{~mm}, 40 \mathrm{~Hz})$. Press ups were completed in a randomised order for a period of $15 \mathrm{~s}$ with 3 min rest. 3D motion capture with synchronous surface electromyography (EMG) of dynamic muscles (triceps, pectoralis major) and stabiliser muscles (serratus anterior, lower trapezius muscles). The introduction of vibration to press ups did not result in kinematic changes to exercises. No muscles analysed displayed significant increases in mean EMG amplitude in response to $30 \mathrm{~L} \mathrm{VIB}$ in the eccentric or the concentric phase of the exercise. $40 \mathrm{H}$ Vibration significantly increased EMG amplitudes of all muscles $(p<$ 0.05 ) except the lower trapezius. Greater increases were observed during the concentric phase of movement. These results indicate that vibration influences muscle activity during press up exercises. However, further studies are required including additional information such as the transmission of vibration to the neck and head, should be completed.
\end{abstract}

Keywords Vibration, EMG, Kinematics, Push Up

\section{Introduction}

To date there have been many studies considering the effect of whole body vibration (WBV) on the lower body, as reviewed by Rehn et al. [1], however yet there have been few investigations on the effects of WBV platforms on the upper body. Hazell et al. [2] considered the effect of WBV during static and dynamic squats and bicep curls on EMG of both the upper and lower body, with results indicating significant increases in signal RMS, yet this was not a consequence of direct vibration of the upper body. In a similar study Marin et al. [3] also reported significant increases in EMG activity of the biceps of older adults following isometric squats and bicep contractions via straps attached to a WBV platform. Gómez-Cabello et al. [4] investigated the effects of 11 week dynamic squats with WBV and found significant increases in both lower and upper body strength. McBride et al. [5] investigated the effect of a direct upper body vibration, via a vibrating dumbbell, on subsequent isometric contractions. The results indicated reduced EMG amplitude with an increased EMG frequency following vibration. Mischi and Cardinale [6] also investigated the influence of vibration during isometric upper limb exercise; reporting an increase in EMG RMS during vibration. However, subsequent muscle function was not analysed, making comparison of results difficult. Tripp et al. [7]considered the influence of a vibrating dumbbell at varying frequencies on elbow joint position sense; the results indicated both increased accuracy and decreased variability. While the investigations reported provide some insight into the influence of vibration on upper limb function, they do not provide an insight into multi-muscular or 'compound' exercises. The press up is a commonly applied exercise to target the upper body for improvements of muscular strength, power and endurance [8]. Investigations of press up exercises have utilised EMG as a tool to examine press ups variations, such as: unstable surfaces e.g. hands placed on Swiss balls or basketball(s), altered hand and/or leg positions and altered hand and/or leg height [9-12]. However, care must be taken when altering the position or style of press up exercises. Modelling of compression forces in the lumbar spine during the various forms of press up have indicated that press up varieties such as amending hand position or speed of movement increases lumbar spine compressive forces by $20-37 \%$, use of unstable surfaces creates increases of $25-55 \%$ and changing completing dynamic 'jumping' style press ups increases lumbar spine forces by 58-238\% [12]. Therefore variations in press up exercises which do not rely on altering the standard position are potentially beneficial. To the best of the author's knowledge, to date there is no published research on the effect of vibration during press ups. The aim of this study is therefore to consider the effect of using WBV platforms on press up exercise performance (posture) and muscular 
activity (EMG). The hypothesis of the investigation is that press ups with vibration will increase the muscular activation of dynamic and stabiliser muscles detected by EMG.

\section{Materials and Methods}

\subsection{Participants}

Nine healthy male participants $(21.1 \pm 3.4$ years, $1.68 \pm$ $0.03 \mathrm{~m}, 72.3 \pm 9.5 \mathrm{~kg}$ ), experienced in recreational training and without any history of recent illness or injury volunteered for the study and provided informed consent in accordance with University Ethics Committee and the Declaration of Helsinki.

\subsection{Study Design}

Three press up conditions were tested on the WBV platform in a randomised cross over study design: press up in the absence of vibration (NVIB), low amplitude and frequency vertical vibration $(30 \mathrm{~L} ; 1.2 \mathrm{~mm}, 30 \mathrm{~Hz}) \mathrm{high}$ amplitude and frequency vertical vibration $(40 \mathrm{H} ; 1.9 \mathrm{~mm}, 40$ $\mathrm{Hz}$ ). Press ups were completed in a randomised order at a self-selected pace by participants for a period of $15 \mathrm{~s}$ with 3 min rest between each set. Participants were assessed for press up ability and familiarisation at least 24 hours before data collection. All press ups were completed in one testing session and all press ups were completed on the platform with feet resting on a bench of equal height to the platform.

\subsection{Data Collection and Processing}

Prior to testing participants completed 4 min arm crank ergometery (Lode Angio, Groningen The Netherlands) as a warm up activity. Press ups were performed with the hands consistently placed at a distance 1.2 times the distance between the acromioclavicular joints. Press ups on a Power Plate Pro 6 whole body vibration platform (Power Plate Ltd). During press ups on the Power Plate the participant's feet were placed on a small bench to replicate the press up position of that on the floor. 3D motion capture was acquired using $16 \mathrm{~mm}$ retroreflective markers on the spine at $\mathrm{C} 7$ and $\mathrm{S} 1$, and acromion processes bilaterally. The sampling frequency of the motion capture was $500 \mathrm{~Hz}$ using 10 Oqus cameras (Qualisys, AB, Sweden). Maximal vertical displacements of the $\mathrm{C} 7$ and $\mathrm{S} 1$ were used to define cervical and pelvic vertical movements, the displacements between the $\mathrm{C} 7$ and $\mathrm{S} 1$ markers were used as a measure of postural changes i.e. kyphotic or lordotic postures. Synchronous to motion capture data surface EMG (DelsysBagnoli system, Boston, USA) was obtained via differential bipolar $\mathrm{Ag}-\mathrm{AgCl}$ electrodes placed on the triceps (TR), pectoralis major (PM), serratus anterior (SA) and lower trapezius (LT) muscles in accordance with SENIAM recommendations [13]. Signal impedance and noise artefacts were minimised by skin preparation and taping of wires to prevent excessive movement during press ups. EMG signals were amplified (1 $\mathrm{k}$ gain) with a bandwidth of $20-450 \mathrm{~Hz}$ and a sampling frequency of $2000 \mathrm{~Hz}$. A single reference electrode was placed on the skin level with the $\mathrm{C} 7$ vertebrae. All synchronous data was acquired using Qualisys track manager software (Qualisys, AB, Sweden) and exported in .c3d format for post processing in Visual3 $\mathrm{D}^{\mathrm{tm}}$ (C-Motion, Inc. Germantown, USA). EMG signals were corrected for zero offset by subtracting the signal mean, fully rectified and smoothed using a $6^{\text {th }}$ order Butterworth low pass filter with a cut-off frequency of $2 \mathrm{~Hz}$. For direct comparison of averaged results between subjects, signals were time normalised from the start to the end of each press up based on the vertical position of the $\mathrm{C} 7$ marker. The mean signal amplitude for both the concentric and eccentric phase was calculated using the vertical displacement of $\mathrm{C} 7$ marker to identify the timing of each phase of the movement. The time normalised signal and mean values were then exported to MatLab (MathWorks, USA) for statistical analysis.

\subsection{Statistical Analysis}

The Anderson-Darling test showed that the kinematic data was not normally distributed, the mean EMG data, with the exception of the lower trapezius during the concentric phase and pectoralis major for the eccentric phase were all normally distributed. Therefore, median values were reported in place of means for the kinematic data [14], with statistical dispersion represented as \pm one median absolute deviation (MAD) for kinematic data and standard error of the means (SEM) were used for mean EMG data. Friedman's test was used to test non-normally distributed conditions and repeated measures ANOVA was used for normally distributed data. The locations of significant differences were identified with Tukey's honest significant difference test to account for repeated measures analysis. In all analyses significance was set at alpha $=0.05$.

For data that was normally distributed the effect size was calculated using $\mathrm{Omega}^{2}\left(\widehat{\omega}^{2}\right)$, data that was not normally distributed the effect size was calculated using the coefficient of concordance $(W)$. The calculations for effect sizes are described in detail in Kinnear and Gray [15].

\section{Results}

The results obtained for all kinematic data during each condition are presented in Table 1. Comparison between the 3 conditions showed no significant differences in the duration of the press ups, vertical displacement of the upper thorax (C7) or pelvis (S1) or spinal posture (C7-S1 distance).

The time normalised EMG amplitude for all muscles analysed is displayed in figure 1.The pattern of muscle activation i.e. the EMG amplitude over time, was consistent in SA and PM. For all muscles the activation pattern for $30 \mathrm{~L}$ was similar to that seen in NVIB. However, the activation patterns for both LT and PM during 40H VIB displays 
differences from NVIB. The LT and PM muscle activation during $40 \mathrm{H}$ displays a new peak emerging in the eccentric phase occurring at approximately halfway through the eccentric phase. The concentric phase of LT $40 \mathrm{H}$ is similar to 30L. In addition to the eccentric phase increase of PM during $40 \mathrm{H}$ VIB, the concentric phase also displays a new peak, again occurring around halfway through the phase.

Table 1. Kinematic parameters for press ups, values are presented as medians \pm 1 MAD.

\begin{tabular}{cccc}
\hline Variable & NVIB & $30 \mathrm{~L}$ & $40 \mathrm{H}$ \\
\hline Duration (s) & $1.76 \pm 0.38$ & $1.69 \pm 0.34$ & $1.76 \pm 0.38$ \\
\hline $\begin{array}{c}\text { C7 vertical } \\
\text { displacement }(\mathrm{m})\end{array}$ & $0.37 \pm 0.05$ & $0.35 \pm 0.07$ & $0.34 \pm 0.04$ \\
\hline $\begin{array}{c}\text { S1 vertical } \\
\text { displacement }(\mathrm{m})\end{array}$ & $0.24 \pm 0.02$ & $0.24 \pm 0.03$ & $0.24 \pm 0.02$ \\
\hline Spinal posture $(\mathrm{m})$ & $0.51 \pm 0.03$ & $0.51 \pm 0.03$ & $0.52 \pm 0.03$ \\
\hline
\end{tabular}

None of the muscles analysed displayed significant increases in mean EMG amplitude in response to 30L VIB in the eccentric or the concentric phase of the exercise. During the eccentric phase the TR muscles displayed a significant increase from NVIB but not from 30L. PM was the only muscle in the eccentric phase with data that was not normally distributed, therefore was analysed with Friedman's test rather than repeated measures ANOVA. The effect size for all muscles was small $(0.2)$, except TR which was medium (0.3).

The mean amplitude of the EMG signals during the concentric phase of the press up exercises displayed significant increases from NVIB in response to $40 \mathrm{H}$ VIB in all muscles except the lower trapezius, where $40 \mathrm{H}$ resulted in a slight decrease in amplitude. LT was the only muscle with data that was not normally distributed and therefore analysed with Friedman's test rather than repeated measures ANOVA. The mean EMG amplitudes for the eccentric and concentric phases are displayed in Figure 2. The effect size for all muscles was small $(0.2)$, except TR which was medium (0.4).

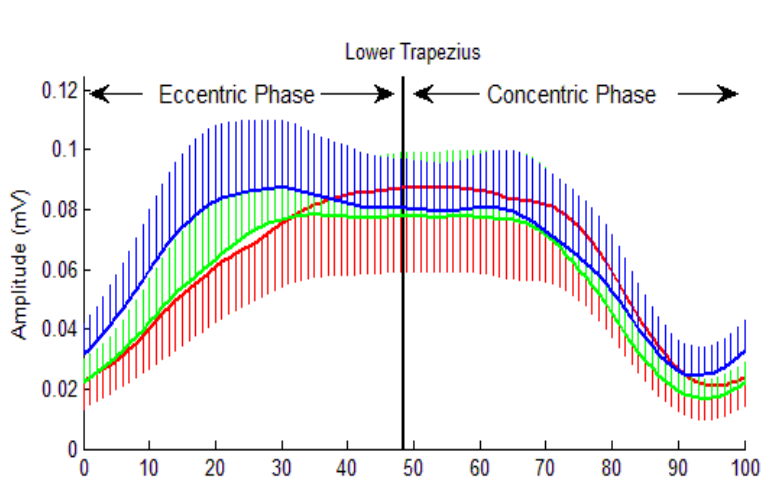

Study 1
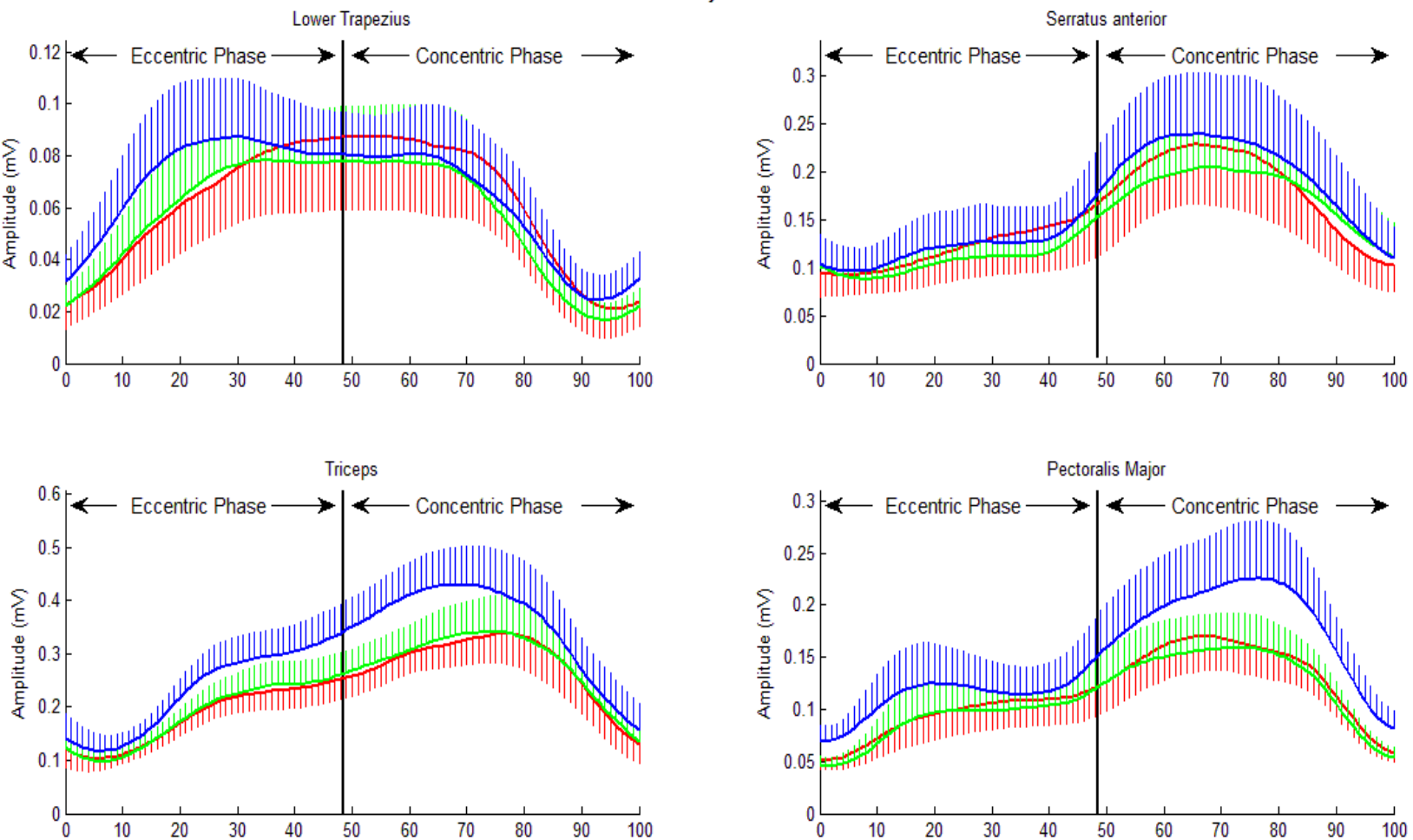

Figure 1. Time normalised EMG amplitude for each muscle during press up exercises. The red line $=\mathrm{NVIB}$, the green line $=30 \mathrm{~L}$ and the blue line $=40 \mathrm{H}$. The error bars represent 1 SEM. 

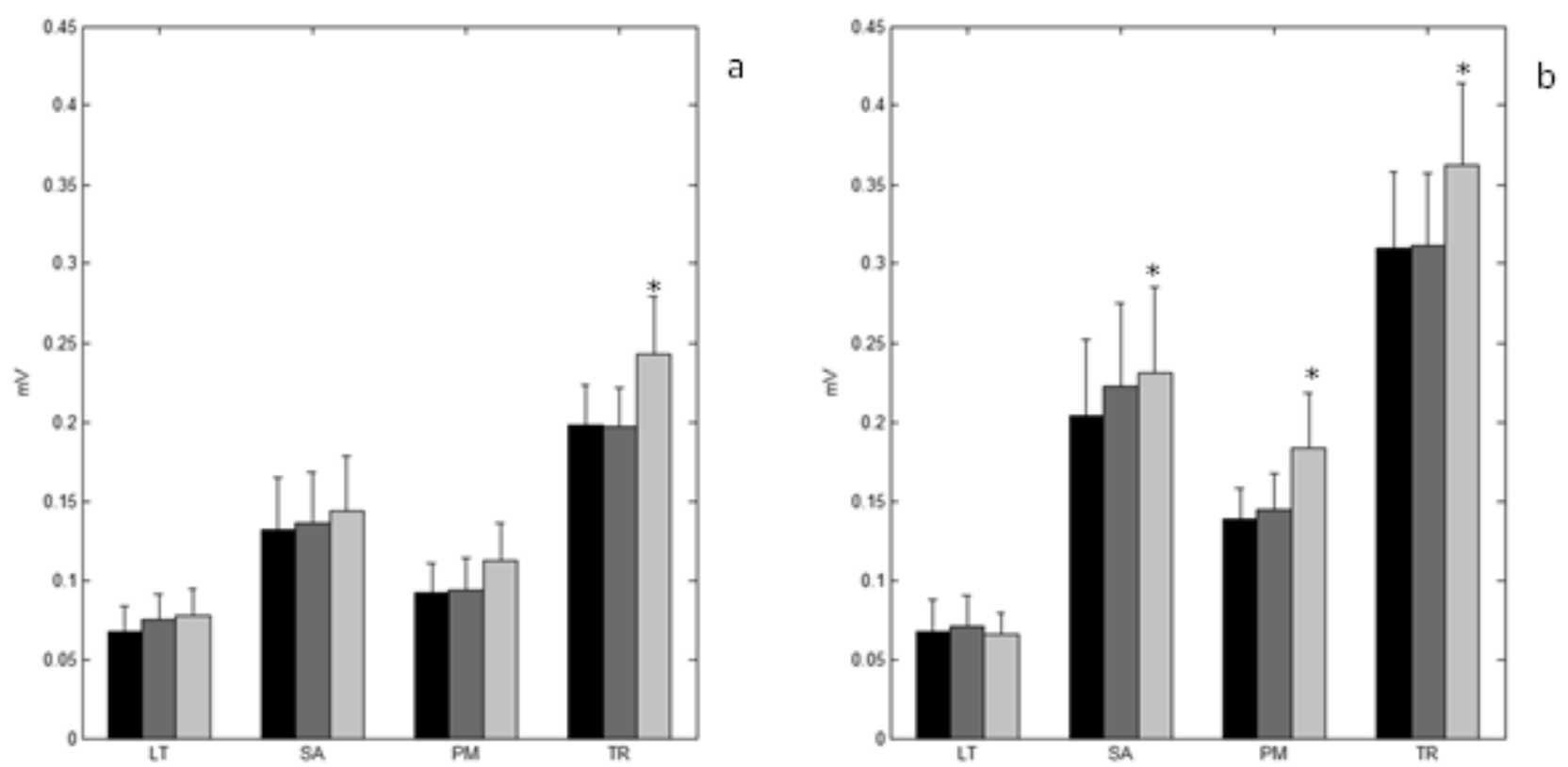

Figure 2. Eccentric phase mean EMG amplitude (a) and Concentric phase mean EMG amplitude (b). The black bars $=$ NVIB, the dark grey bars $=30 \mathrm{~L}$ and the light grey bars $=40 \mathrm{H}$. The error bars $=1 \mathrm{SEM}, *=$ significantly different from NVIB $(\mathrm{p}<0.05)$.

\section{Discussion}

The lack of significant difference between the timing to complete press ups, vertical displacements of cervical spine (C7) and pelvis (S1) and the distance between C7 and S1 indicate that the participants performed each press up in the same pace, range of motion and did not change posture i.e. develop kyphotic or lordotic postures during the press ups in any condition. This consistency of movements is important for two reasons, firstly vibration has previously been shown to reduce postural stability [16] and secondly the consistency in movement removes the variable of changing exercise performance when considering changes in EMG. Care was taken during the initial set up of NVIB, $30 \mathrm{~L}$ and $40 \mathrm{H}$ to ensure all participants were positioned with hands and feet level, as changing the relative position of height and hands and feet has been shown to influence EMG activity of shoulder musculature, particularly that of the serratus anterior $[11,17]$. In addition, hand position was standardised as the distance equivalent to that between the acromioclavicular distance with avoidance of movement in a caudad and cephalad direction, as this has been shown to influence the EMG activity of the shoulder and upper arms agonist muscles $[9,18]$. During a standard press up it has previously been reported that the forces experienced are typically around two thirds of body weight $[9,19]$. Although it has been shown that narrow base press ups, i.e. with the hands closer together, have an elbow torque which is $71 \%$ of maximal torque, compared to wide base press ups which are only $29 \%$ [20] which likely explains the reported increase in EMG activity during press ups with altered hand positions $[9,18]$. Considering the adherence to specific participant positional set up, combined with the lack of significant differences in kinematic parameters, it can be assumed the only variable influencing muscle performance was the introduction of vibration. WBV platforms have been shown to increase EMG activity of the lower body [21-23] with results indicating that increases occur in line with that obtained during increasing resistance of comparable exercises [24]. Utilising muscle contractions typical of the activity to be analysed i.e. without the intervention condition, has also been shown to have the advantage of providing a form of representative activation of the position for the movement to be analysed [9], therefore providing comparable muscle activity for analysis. The results in Figure 1 and Figure 2 provide a sense of the level of increase in activity resulting from changing the vibration experienced during press up exercises. The greatest increases observed in EMG amplitude were observed in TR during $40 \mathrm{H} \mathrm{VIB,} \mathrm{with}$ increases apparent during both the eccentric and concentric phases. Interestingly Marin et al. [25] found that elbow extension during WBV is enhanced with high magnitude vibration $(50 \mathrm{~Hz}, 2.5 \mathrm{~mm})$ but not low vibration $(30 \mathrm{~Hz}, 1.2$ $\mathrm{mm}$ ), suggesting that triceps muscles require a certain threshold of vibration to enhance performance. The pattern of EMG activity for PM displayed a significant change in mean activation during $40 \mathrm{H}$ VIB only during the concentric phase. However, it should be noted that the data distribution of the mean EMG amplitude data during the eccentric phase was not normally distributed and therefore the analysis via non-parametric methods did not have the same power as that used to analyse the other muscles during the eccentric phase. The low and medium effect sizes obtained for the results are a further indication that tests with reduced power i.e. non-parametric hypothesis tests, are less likely to able to confirm statistical significance. The eccentric phase of PM 
displayed a change in the pattern of activation, with a new peak becoming apparent at approximately halfway through the eccentric phase (see Figure 1). An increase in LT activation was also noted at this approximate time point, suggesting potential for increased muscular demand at this point of the movement. To the best of the authors knowledge there is no data on transmission of vibration through the human body in this position; yet it has previously been shown that when standing with increased knee flexion reduces vertical transmission of vibration [26]. It is therefore a reasonable assumption that whilst the elbows are flexed there is increased demand for damping of vibration transmission via the shoulder girdle, though further studies should be completed to confirm this. While patterns of EMG activity in LT did occur it should be noted that the increases in mean amplitude were not significant. The LT is the furthest analysed muscle from the WBV platform surface and is the only muscle to not display any significant increases in mean EMG amplitude. This observation can potentially be explained by the postulation that increased distance from the platform decreases muscle activity [27] indicating the role of the muscle and the location of the muscle are the key factors in the influence of vibration on muscle activity. This theory is given further credence by previously reported values of EMG during standard press ups to fatigue, where the SA displayed changes 3.5 times the level of the lower trapezius [28]. These differences in activation level are also potentially explained by the fact that the SA has previously been reported to be both the prime mover of the scapula [29] and a stabiliser muscle, particularly in prevention of conditions such as scapula winging [30].

One final area worthy of note is that it has previously been reported that large differences have been observed between skilled and unskilled performers of press up exercises [12]. Though unfortunately while the timing of EMG activity was also reported, a single pass Butterworth filter was applied to the signals. Single pass filters cause phase distortions resulting in time lags within the signal [31] and are therefore not comparable to the data obtained within this investigation.

\section{Conclusion}

The introduction of vibration to press up exercises does not result in kinematic changes to exercise performance. Duration, posture and range of motion did not change in any tested condition, indicating the actual exercise performance did not change in any condition. In contrast EMG activity displayed highly significant differences, particularly in the shoulder stabiliser muscles which displayed increases of 3.5 times the activity in a standard press up. In addition, the pattern of EMG activity in both pectoralis major, serratus anterior and lower traps displayed significant changes. These results suggest that vibration has significant influence on both dynamic and stabilising muscles of the shoulder; these changes are potentially influenced by the changing the force moment arm during the movement. Use of the information in this pilot study can be used to determine the statistical power of sample sizes of future investigations in to the effect of vibration on press up exercises. Prior to recommendations being made for the application of vibration in press up exercises further studies should also be completed on the transmission of vibration through the upper body and the potential safety implications of transmission of vibration to the neck and head.

\section{REFERENCES}

[1] Rehn B, Lidström J, Skoglund J, et al. Effects on leg muscular performance from whole-body vibration exercise: a systematic review. Scandinavian journal of medicine \& science in sports 2007;17:2-11. doi:10.1111/j.1600-0838.2006.00578.x

[2] Hazell TJ, Jakobi JM, Kenno KA. The effects of whole-body vibration on upper- and lower-body EMG during static and dynamic contractions. Applied physiology, nutrition, and metabolism 2007;32:1156-63. doi:10.1139/H07-116

[3] Marín PJ, Santos-Lozano A, Santin-Medeiros F, et al. Whole-body vibration increases upper and lower body muscle activity in older adults: potential use of vibration accessories. Journal of electromyography and kinesiology 2012;22:456-62. doi:10.1016/j.jelekin.2012.02.003

[4] Gómez-Cabello A, González-Agüero A, Ara I, et al. Effects of a short-term whole body vibration intervention on physical fitness in elderly people. Maturitas 2013;74:276-8. doi:10.1016/j.maturitas.2012.12.008

[5] McBride J, Porcari J, Scheunke M. Effect of vibration during fatiguing resistance exercise on subsequent muscle activity during maximal voluntary isometric contractions. The Journal of Strength \& Conditioning Research 2004;18:777-81.

[6] Mischi M, Cardinale M. The effects of a $28-\mathrm{Hz}$ vibration on arm muscle activity during isometric exercise. Medicine and science in sports and exercise 2009;41:645-53. doi:10.1249/MSS.0b013e31818a8a69

[7] Tripp B, Faust D, Jacobs P. Elbow joint position sense after neuromuscular training with handheld vibration. Journal of athletic training 2009;44:617-23. doi:10.4085/1062-6050-44.6.617

[8] Contreras B, Schoenfeld B, Mike J, et al. The Biomechanics of the Push-up: Implications for Resistance Training Programs. Strength and Conditioning Journal 2012;34:41-6.

[9] Gouvali M, Boudolos K. Dynamic and electromyographical analysis in variants of push-up exercise. J Strength Cond Res 2005;19:146-51.

[10] Lehman GJ, MacMillan B, MacIntyre I, et al. Shoulder muscle EMG activity during push up variations on and off a Swiss ball. Dynamic medicine?: DM 2006;5:7. doi:10.1186/1476-5918-5-7

[11] Lehman GJ, Gilas D, Patel U. An unstable support surface does not increase scapulothoracic stabilizing muscle activity 
during push up and push up plus exercises. Manual therapy 2008;13:500-6. doi:10.1016/j.math.2007.05.016

[12] Freeman S, Karpowicz A, Gray J, et al. Quantifying muscle patterns and spine load during various forms of the push-up. Medicine and science in sports and exercise 2006;38:570-7. doi:10.1249/01.mss.0000189317.08635.1b

[13] Hermens HJ, Freriks B, Disselhorst-Klug C, et al. Development of recommendations for SEMG sensors and sensor placement procedures. Journal of electromyography and kinesiology 2000;10:361-74.

[14] Lang T, Secic M. How to Report Statistics in Medicine: Annotated Guidelines for Authors, Editors and Reviewers. Second Edi. American College of Physicians 2006.

[15] Kinnear PR, Gray CD. PASW 17 Statistics made simple. 2010.

[16] Lee B-C, Martin BJ, Sienko KH. Directional postural responses induced by vibrotactile stimulations applied to the torso. Experimental brain research ExperimentelleHirnforschungExpérimentationcérébrale 2012;222:471-82. doi:10.1007/s00221-012-3233-2

[17] Lear LJ, Gross MT. An electromyographical analysis of the scapular stabilizing synergists during a push-up progression. The Journal of orthopaedic and sports physical therapy 1998;28:146-57.

[18] Cogley R, Archambault T, Fibeger J, et al. Comparison of Muscle Activation Using Various Hand Positions During the Push-Up. The Journal of Strength and Conditioning Research 2005;19:628-33.

[19] Blackard D, Jensen R, Ebben W. Use of EMG analysis in challenging kinetic chain terminology. Medicine \& Science in Sports \& Exercise 1999;31:443-8. doi:10.1097/00005768-199903000-00014

[20] Donkers MJ, An K-N, Chao EYS, et al. Hand position affects elbow joint load during push-up exercise. Journal of Biomechanics doi:10.1016/0021-9290(93)90026-B $1993 ; 26: 625-32$.

[21] Eckhardt H, Wollny R, Müller H, et al. Enhanced myofiber recruitment during exhaustive squatting performed as whole-body vibration exercise. The Journal of Strength \& Conditioning Research 2011;25:1120-5.
[22] Ritzmann R, Gollhofer A, Kramer A. The influence of vibration type, frequency, body position and additional load on the neuromuscular activity during whole body vibration. European journal of applied physiology 2013;113:1-11.

[23] Hazell T, Kenno K, Jakobi J. Evaluation of muscle activity for loaded and unloaded dynamic squats during vertical whole-body vibration. The Journal of Strength \& Conditioning Research 2010;24:1860-5.

[24] Marín PJ, Santos-Lozano A, Santin-Medeiros F, et al. A comparison of training intensity between whole-body vibration and conventional squat exercise. Journal of electromyography and kinesiology 2011;21:616-21. doi:10.1016/j.jelekin.2010.12.008

[25] Marin PJ, Azael h, Nuria S, et al. Effects of Different Magnitudes of Whole-Body Vibration on Arm Muscular Performance. Journal of Strength \& Conditioning Research 2010;24:2506-11.

[26] Abercromby A, Amonette W, Layne C, et al. Vibration exposure and biodynamic responses during whole-body vibration training. Medicine and science in sports and exercise 2007;39:1794-800. doi:10.1249/mss.0b013e3181238a0f

[27] Wirth B, Zurfluh S, Müller R. Acute effects of whole-body vibration on trunk muscles in young healthy adults. Journal of electromyography and kinesiology?: official journal of the International Society of Electrophysiological Kinesiology 2011;21:450-7. doi:10.1016/j.jelekin.2010.12.007

[28] Borstad JD, Szucs K, Navalgund A. Scapula kinematic alterations following a modified push-up plus task. Human movement science 2009;28:738-51. doi:10.1016/j.humov.2009.05.002

[29] Dvir Z, Berme N. The shoulder complex in elevation of the arm: A mechanism approach. Journal of Biomechanics 1978;11:219-25. doi:10.1016/0021-9290(78)90047-7

[30] Kendall F, McCreary E, Provance P. Muscles Testing and Function, with Posture and Pain. Fourth Edi. Lippincott Williams and Wilkins 1993.

[31] Winter D. Biomechanics and Motor Control of Human Movement. Third Edit. John Wiley and Sons,Inc. 2005. 